\title{
Fast $k$ Most Similar Neighbor Classifier for Mixed Data Based on a Tree Structure and Approximating-Eliminating
}

\author{
Selene Hernández-Rodríguez, J.A. Carrasco-Ochoa, \\ and J. Fco. Martínez-Trinidad \\ Computer Science Department \\ National Institute of Astrophysics, Optics and Electronics \\ Luis Enrique Erro No. 1, Sta. María Tonantzintla, Puebla, CP: 72840, México \\ \{selehdez, ariel, fmartine\} @ccc. inaoep.mx
}

\begin{abstract}
The $k$ nearest neighbor $(k-N N)$ classifier has been extensively used as a nonparametric technique in Pattern Recognition. However, in some applications where the training set is large, the exhaustive $k-N N$ classifier becomes impractical. Therefore, many fast $k-N N$ classifiers have been developed to avoid this problem. Most of these classifiers rely on metric properties, usually the triangle inequality, to reduce the number of prototype comparisons. However, in soft sciences, the prototypes are usually described by qualitative and quantitative features (mixed data), and sometimes the comparison function does not satisfy the triangle inequality. Therefore, in this work, a fast $k$ most similar neighbor $(k-M S N)$ classifier, which uses a Tree structure and an Approximating and Eliminating approach for Mixed Data, not based on metric properties (Tree AEMD), is introduced. The proposed classifier is compared against other fast $k-N N$ classifiers.
\end{abstract}

Keywords: k-NN Classifier, Fast k-NN Classifiers, Mixed Data.

\section{Introduction}

The $k-N N$ [1] classifier has been extensively used as a nonparametric technique in Pattern Recognition. In order to decide the class of a new prototype, the $k$ - $N N$ classifier performs an exhaustive comparison between the prototype to classify (query) and the prototypes in the training set $(T)$. However, when $T$ is large, the exhaustive comparison is expensive. To avoid this problem, many fast $k-N N$ classifiers have been developed. Some of these classifiers are exact methods, because they find the same $k$ $N N$ that would be found using the exhaustive search. Some others are approximate methods, because they do not guarantee to find the $k-N N$ to a query prototype among the training set, but they find an approximation faster than the exact methods.

To avoid prototype comparisons during the search of the $k-N N$, different approaches such as: approximating-eliminating [2-6] and tree-based [5-9] have been developed. 
Tree-based classifiers consist of two phases: preprocessing and classification. In the preprocessing phase, the prototypes in $T$ are organized in a tree structure. In the classification phase, the tree is traversed to find the $k-N N$. The speed up is obtained while the exploration of some parts of the tree is avoided, applying pruning rules derived from the triangle inequality property. One of the first fast $k-N N$ classifiers, that uses a tree structure, was proposed by Fukunaga and Narendra [7]. Some improvements, over the pruning rule used in the Fukunaga and Narendra's (FN) classifier, have been proposed by Moreno-Seco et al. (MS classifier) [8] and Oncina et al. (ONC classifier) [9].

The first classifier based on the approximating-eliminating approach was AESA (Approximating Eliminating Search Algorithm), proposed by Vidal [2]. Some improvements over AESA, have been developed; for example: LAESA [3], iAESA [4], probabilistic iAESA [4], TLAESA [5] and ModTLAESA [6].

All these classifiers were designed to work with quantitative data when the prototype comparison function satisfies the triangle inequality. However, in soft sciences as Medicine, Geology, Sociology, etc., the prototypes are commonly described by mixed data. In these cases, sometimes the comparison function does not satisfy the triangle inequality and therefore, we can not use most of the classifiers proposed for quantitative prototype descriptions. Thus, if a metric is not available but a comparison function that evaluates the similarity between a pair of prototypes could be defined, the objective would be to find the $k$ most similar neighbors $(k-M S N)$ and use them for classifying. For this reason, in this paper we introduce a fast approximate $k-M S N$ classifier, based on a tree structure and a new approximating and eliminating approach for mixed data (Tree AEMD). The aim of our algorithm is not to find the exact $k-M S N$ but fast finding an approximation of them, which allows obtaining classification accuracy close to that obtained by the exact ones.

This paper is organized as follows: in Section 2 the comparison function used in this work is described. In Section 3 Tree AEMD is introduced. Finally, we report experimental results in Section 4 and conclusions in Section 5.

\section{Comparison Functions for Mixed Data}

In this work, the function $D$ [10], which does not fulfil the triangle inequality and allow us to compare prototypes described by mixed data, was used. Let us consider a set of prototypes $\left\{P_{1}, P_{2}, \ldots, P_{N}\right\}$, each of them described by $d$ attributes $\left\{x_{1}, x_{2}, \ldots\right.$, $\left.x_{d}\right\}$. Each feature could be quantitative or qualitative. The function $D$ is defined as follows:

$$
D\left(P_{1}, P_{2}\right)=1-\frac{\left|\left\{x_{i} \mid C_{i}\left(x_{i}\left(P_{1}\right), x_{i}\left(P_{2}\right)\right)=1\right\}\right|}{d}
$$

For qualitative data $C_{i}\left(x_{i}\left(P_{1}\right), x_{i}\left(P_{2}\right)\right)$ is the following:

$$
C_{i}\left(x_{i}\left(P_{1}\right), x_{i}\left(P_{2}\right)\right)=\left\{\begin{array}{cc}
1 & \text { If } x_{i}\left(P_{1}\right)=x_{i}\left(P_{2}\right) \text { and neither } x_{i}\left(P_{1}\right) \text { nor } x_{i}\left(P_{2}\right) \\
& \text { is a missing value } \\
0 & \text { otherwise }
\end{array}\right.
$$


For quantitative data $C_{i}\left(x_{i}\left(P_{1}\right), x_{i}\left(P_{2}\right)\right)$ is the following:

$$
C_{i}\left(x_{i}\left(P_{1}\right), x_{i}\left(P_{2}\right)\right)=\left\{\begin{array}{cc}
1 & \text { If }\left|x_{i}\left(P_{1}\right)-x_{i}\left(P_{2}\right)\right|<\sigma_{i} \text { and neither } x_{i}\left(P_{1}\right) \operatorname{nor} x_{i}\left(P_{2}\right) \\
& \text { is a missing value } \\
0 & \text { otherwise }
\end{array}\right.
$$

Where, $\sigma_{i}$ is the standard deviation of the attribute $x_{i}$ in $T$. In this work, the Similarity $S\left(P_{1}, P_{2}\right)=1-D\left(P_{1}, P_{2}\right)$ was used for our algorithm.

\section{Proposed Classifier}

The proposed classifier (Tree AEDM) consists of two phases: preprocessing and classification, which are described in the next subsections.

\subsection{Preprocessing Phase}

In the preprocessing phase, a tree structure, a similarity matrix, a representative prototype per class and a similarity threshold are computed as follows:

1. Tree structure $(T S)$ : This is created using the prototypes in $T$. In this case, the training set is hierarchically decomposed to create the tree structure. For the decomposition, the C-Means with Similarity Functions algorithm (CMSF) [10], which is an extension of the $C$-Means algorithm to work with mixed data and any prototype comparison function, is used. In the original C-Means the mean of the prototypes of a cluster is considered as the centre of it, meanwhile in $C M S F$ a representative prototype in the sample is used as the centre of the cluster. See [10] for details.

The node 0 (root of $T S$ tree) contains the whole training set. In order to create the following levels of the tree, each node $p$ of the tree is divided in $C$ clusters, in such a way that each cluster represents a descendant node of $p$. Each node $p$ of the tree contains three features which are: $S_{p}$ the set of prototypes that belong to $p ; N_{p}$ the number of prototypes in $p$ and $\operatorname{Rep}_{p}$ a representative prototype of $p$, which is the most similar in average to the prototypes contained in the node.

Each node is divided again and this process is repeated until a stop criterion is fulfilled. In this work, we used a stop criterion proposed in [11]. This criterion takes into account not only the number of prototypes in the node, but also the class distribution of these prototypes. In this case, if certain percentage (PercThres) of the prototypes in a cluster belongs to the same class, two nodes are created, for replacing the original one. Using the prototypes that belong to the majority class, a leaf node is created and marked with the majority class. The remaining prototypes are assigned to a second node. In the second node, the size is considered to decide if the node is a leaf (if $N_{p} \leq$ $N o P$ ) or if the node will be divided again. In the nodes where a majority class, can not be recognized, only the size of the nodes is considered to create leaves (if $N_{p} \leq N o P$ then the node is a leaf).

2. Similarity matrix $(S M)$. A matrix containing the similarities per attribute between all the representative prototypes of the nodes in the tree is computed. In this case, $\operatorname{SM}\left(\operatorname{Rep}_{a}, \operatorname{Rep}_{b}, x_{i}\right)=1$ if, according to certain criterion, we can conclude that the representative prototypes $\operatorname{Rep}_{a}$ and $\operatorname{Rep}_{b}(a, b=1, \ldots, N o N o d$; NoNod is the number of nodes in the tree) are similar considering the attribute $x_{i}(i=1, \ldots, d)$ and 
$\operatorname{SM}\left(\operatorname{Rep}_{a}, \operatorname{Rep}_{b}, x_{i}\right)=0$, otherwise. In this work, the similarity criteria described in Section 2, were used.

The required space to store SM is NoNod x NoNod $\mathrm{x} d$ but each element is a bit, therefore, NoNod x NoNod words of $d$ bits are needed for storing SM.

3. A representative prototype per class $\left(R P_{c}\right)$. Taking advantage of the class information, in order to obtain a first approximation during the classification phase, we propose to use a representative prototype per class $\left(R P_{c}\right)$. Let Class $_{c}$ be the set of prototypes in $T$, belonging to the class $c$. Then, for each prototype $P_{a} \in$ Class $_{c}$, the average similarity between $P_{a}$ and the remaining prototypes belonging to the same class is computed as follows:

$$
\operatorname{AvgSim}\left(P_{a}\right)=\frac{\sum_{P_{b} \in \text { Class }_{c}, b \neq a} S\left(P_{a}, P_{b}\right)}{\mid \text { Class }_{c} \mid-1}
$$

In this way, the representative prototype for the class $c\left(R P_{c}\right)$ will be the one most similar on average:

$$
R P_{c}=\operatorname{ArgMax}\left(\operatorname{AvgSim}\left(P_{a}\right)\right), \quad \forall P_{a} \in \operatorname{Class}_{c}
$$

This process is repeated for every $c=1, \ldots, N C$, where $N C$ is the number of classes in the training set.

4. Similarity threshold between prototypes (SimThres). This value is used as a confidence threshold to make decisions during the classification phase. In order to obtain SimThres, for each class $c$, the average value of similarity among the prototypes belonging to the same class is computed as follows:

$$
\operatorname{ClassAvgSim}(c)=\frac{\sum_{P_{a} \in \text { Class }_{c}} \operatorname{AvgSim}\left(P_{a}\right)}{\mid \text { Class }_{c} \mid}
$$

Finally, the similarity threshold (SimThres) is computed as the average value of ClassAvgSim $(c)$, for all $c=1, . ., N C$.

\subsection{Classification Phase of Tree AEDM}

Given a new prototype $Q$ to classify, $T S, S M, R P_{c}$ and SimThres, computed during the preprocessing phase, are used to avoid prototype comparison. In this phase, a classification algorithm, using new approximating and eliminating steps (which are not based on the triangle inequality), is proposed as follows:

1. Initial approximation step. At the beginning of the algorithm, the prototype $Q$ is compared against the representative prototypes $\left(R P_{c}, c=1, \ldots, N C\right)$ to obtain a first approximation to the $M S N$ :

$$
M S N=\operatorname{ArgMin}\left(D\left(Q, R P_{c}\right)\right), c=1, \ldots, N C
$$

2. Tree traversal algorithm. In order to update the $k-M S N$, the tree structure is traversed. Two algorithms to traverse the tree are proposed:

- Tree traversal algorithm, using Depth First Search (DFS).

- Tree traversal algorithm, using Best First Search (BFS). 
3. Classification step. Finally, the majority class of the $k-M S N$ is assigned to $Q$.

In the next subsections, the tree traversal algorithms are presented.

\subsubsection{Tree Traversal Algorithm, Using DFS}

\section{CurrentNode=root}

2. Approximating step: All the direct descendant nodes ( $n$ ) of CurrentNode, neither traversed nor eliminated by the Eliminating step, are compared to $Q$, in order to select a new node (MSNod) to continue the tree traversal:

$$
M S N o d=\operatorname{ArgMin}\left(D\left(Q, \operatorname{Rep}_{n}\right)\right)
$$

The list of the $k-M S N$ is updated during the tree traversal, MSNod is marked as already traversed and CurrentNode $=$ MSNod.

3. If $D\left(Q, R_{e} p_{M S N o d}\right) \geq$ SimThres, go to step 4, in other case, go to step 5 .

The elimination step will be applied only if the current $\operatorname{Rep}_{M S N o d}$ is similar enough to $Q$, which is determined using SimThres.

4. Eliminating step: Since $\operatorname{Rep}_{M S N o d}$ is very similar to $Q$, if a prototype is not similar to $\operatorname{Rep}_{M S N o d}$ in at least the same attributes where $\operatorname{Rep}_{M S N o d}$ is similar to $\mathrm{Q}$, that prototype will not be more similar to $Q$ than $\operatorname{Rep}_{M S N o d}$. Therefore, the representative prototype of MSNod $\left(\operatorname{Rep}_{M S N o d}\right)$ is used to eliminate nodes of the tree. In this step, BR containing the similarity per attribute, between $Q$ and $\operatorname{Rep}_{M S N o d}$, is created as follows:

$$
B R_{i}\left(Q, \operatorname{Rep}_{M S N o d e}\right)=C_{i}\left(x_{i}(Q), x_{i}\left(\operatorname{Rep}_{M S N o d e}\right)\right), i=1, . ., d
$$

Thus, $B R_{i}\left(Q, \operatorname{Rep}_{M S N o d}\right)=1$ if $Q$ and $\operatorname{Rep}_{M S N o d}$ are similar in the attribute $x_{i}$ and $B R_{i}\left(Q, \operatorname{Rep}_{M S N o d}\right)=0$, in other case. Using $B R$, and $S M$ matrix, those nodes $n$ in the tree (not yet traversed or eliminated), having a representative prototype $\left(\operatorname{Rep}_{n}\right)$, which is not similar to $\operatorname{Rep}_{M S N o d}$, at least in the same attributes where $\operatorname{Rep}_{M S N o d}$ is similar to $Q$, are eliminated.

For example, suppose that $R e p_{1}, \operatorname{Rep}_{2}, Q$ and $\operatorname{Rep}_{M S N o d}$, are such that $B R(Q$, $\left.\operatorname{Rep}_{M S N o d}\right)=[1,1,0,1,1,1,0,0], S M\left(\operatorname{Rep}_{M S N o d}, \operatorname{Rep}_{1}\right)=[1,1,1,1,1,1,0,1]$ and $S M\left(\operatorname{Rep}_{M S N o d}\right.$, $\left.\operatorname{Rep}_{2}\right)=[1,0,0,0,0,1,0,1]$. Then, according to this criterion, $\operatorname{Rep}_{1}$ is not eliminated because it is similar to $\operatorname{Rep}_{M S N o d}$ in the same attributes, where $\operatorname{Rep}_{M S N o d}$ is similar to $Q$ (attributes 1, 2, 4, 5 and 6). But $\operatorname{Rep}_{2}$ is eliminated, because $\operatorname{Rep}_{2}$ is not similar in the same attributes, where $R e p_{M S N o d}$ is similar to $Q$ (Rep $_{M S N o d}$ is similar to $Q$ in attribute 2 , but $\operatorname{Rep}_{2}$ is not similar to $\operatorname{Rep}_{M S N o d}$ in this attribute). The similarity per attribute between $\operatorname{Rep}_{M S N o d}$ and $\operatorname{Rep}_{1}\left(S M\left(\operatorname{Rep}_{M S N o d}, \operatorname{Rep}_{1}\right)\right)$ and between $\operatorname{Rep}_{M S N o d}$ and $\operatorname{Rep}_{2}$ $\left(S M\left(\operatorname{Rep}_{M S N o d}, \operatorname{Rep}_{2}\right)\right)$ are known, since these similarities were computed in the preprocessing phase. The similarity between $\operatorname{Rep}_{M S N o d}$ and $Q$, has already been computed and stored in $B R$.

5. If CurrentNode is a leaf, then go to step 6; in other case, go to step 2.

6 . When a leaf node $l$ is reached:

- If $l$ has been marked with the majority class, given that, most of the times the most similar prototype in the node will be in the majority class, and will be close to the representative prototype, only the representative prototype $\operatorname{Rep}_{l}$ is considered to update the list of $k-M S N$.

- If $l$ has not been marked with the majority class, then, as there are few prototypes in the node $\left(N_{p} \leq N o P\right)$, an exhaustive comparison between $Q$ and the prototypes contained in $l$ is done, for updating the list of $k-M S N$. 
7. The tree traversal gets back one level of the tree to evaluate the remaining nodes.

8. If there are still neither traversed nor eliminated nodes in the tree, go to step 9 . In other case, go to step 10 .

9. Assign to CurrentNode the most similar node to $Q$ in the corresponding level and go to step 5.

10. End of the algorithm.

\subsubsection{Tree Traversal Algorithm, Using BFS}

\section{CurrentNode $=$ root, $L=\varnothing$.}

2. Approximating step: All the direct descendant nodes of CurrentNode, not yet traversed or eliminated by the Eliminating step, are compared to $Q$ and added to the list $L$, which is sorted in such a way that the most similar node to $Q$ is in the first place $(L(1)=M S N o d)$. This node $(M S N o d)$ is selected to continue the tree traversal. The node $L(1)$ is eliminated from the list (because, it is already traversed). The $k-M S N$ is updated. The node MSNod is marked as node already traversed and CurrentNode $=$ MSNod .

3. If $D\left(Q\right.$, Rep $\left._{M S N o d}\right) \geq$ SimThres, go to step 4, in other case, go to step 5 .

4. Eliminating step: this step is the same described for the tree traversal algorithm, using DFS (step 4, section 3.2.1).

5. If CurrentNode is a leaf, then go to step 6; otherwise, go to step 2.

6 . When a leaf node $l$ is reached; this step is the same described for the tree traversal algorithm, using DFS (step 6, section 3.2.1).

7. If the list $L$ is not empty; then go to step 8. Otherwise, go to step 10.

8. If the first element in the list $L$ has not been eliminated yet, then go to step 9 . In other case, eliminate this element from the list and go to step 7.

9. CurrentNode $=L(1)$ and go to step 5 .

10. End of the algorithm.

\section{Experimental Results}

In order to evaluate Tree AEMD classifier, it was compared against the exhaustive $k$ $N N$ [1], FN [7], MS [8], ONC [9], AESA [2], LAESA $(|B P|=20 \%$ of the prototypes in the dataset) [3], iAESA [4], Probabilistic iAESA (using 70\% as percentage threshold of the data set) [4], TLAESA [5] and ModTLAESA [6] classifiers. The comparison function $D$, described in Section 2 was used.

For the experiments, 10 datasets from the UCI repository [12] were used (4 mixed datasets: Hepatitis, Zoo, Flag and Echocardiogram. 3 qualitative datasets: Hayes, Soybean-large and Bridges. 3 quantitative: Glass, Iris and Wine).

In order to compare the different classifiers, the accuracy (Acc) and the percentage of comparisons between prototypes (Comp), were considered. The accuracy was computed as follows:

$$
A c c=\frac{\text { NoCorrectObj }}{\text { NoTestObj }}
$$

Where, NoCorrectObj is the number of correctly classified prototypes in the test set and NoTestObj is the size of the test set. The percentage of comparisons between prototypes was computed as follows: 


$$
\text { Comp }=\frac{\text { NoCompFastClass } * 100}{\text { NoTrainingObj }}
$$

Where, NoCompFastClass is the number of comparisons done by the fast classifier, and NoTrainingObj is the size of the training set. According to (12), for the exhaustive classifier, the $100 \%$ of the comparisons is done.

In all the experiments, $k=1$ (for $k-M S N$ ) was used. In [11] different experiments for choosing a value of the parameter $C$ and PercThres were done. In our experiments, $C=3, N o P=20$, and PercThres $=100 \%$ were used, since in [11], the fast classifiers reached their best results with these values.

In tables 1 and 2, the corresponding results (Acc and Comp) obtained with the different classifiers, are shown. From these tables, we can observe that when the comparison function does not satisfy the triangle inequality, FN, ONC, AESA, LAESA, iAESA, TLAESA and ModTLAESA classifiers become approximate methods. However, the percentage of comparisons is, on average, reduced from $100 \%$, done by the exhaustive search, to $58.17 \%(\mathrm{FN}), 33.79 \%$ (ONC), $53.47 \%$ (TLAESA), $30.10 \%$ (ModTLAESA), $28.79 \%$ (LAESA), $25.82 \%$ (AESA) and 23.88\% (iAESA). The classifier proposed in this work (Tree AEMD), did the smallest number of prototype comparisons, using DFS (9.39\%) and BFS (8.81\%).

The experiments were repeated, using $k=3$ and $k=5$ and the performance of the classifiers was similar.

Table 1. Obtained results using different classifiers

\begin{tabular}{|c|c|c|c|c|c|c|c|c|c|c|c|c|}
\hline \multirow[t]{2}{*}{ Datasets } & \multicolumn{2}{|c|}{$\begin{array}{l}\text { Exhaustive } k \text { - } \\
\text { NN classifier }\end{array}$} & \multicolumn{2}{|c|}{$\mathrm{FN}$} & \multicolumn{2}{|c|}{ MS } & \multicolumn{2}{|c|}{ ONC } & \multicolumn{2}{|c|}{ AESA } & \multicolumn{2}{|c|}{ LAESA } \\
\hline & $A C C$ & Comp & $A C C$ & Comp & $A C C$ & Comp & $A C C$ & Comp & $A C C$ & Comp & $A C C$ & Comp \\
\hline Hep. & 81,66 & 100 & 82,00 & 132,9 & 81,36 & 75,98 & 80,71 & 68,59 & 80,57 & 52,96 & 80,54 & 61,86 \\
\hline Zoo & 96,00 & 100 & 95,41 & 47,53 & 94,35 & 35,44 & 94,18 & 23,90 & 96,00 & 23,50 & 96,00 & 15,26 \\
\hline Flag & 54,67 & 100 & 52,26 & 48,26 & 50,21 & 45,93 & 50,39 & 32,98 & 51,45 & 28,02 & 51,45 & 25,73 \\
\hline Echo. & 82,44 & 100 & 81,60 & 103,8 & 80,15 & 85,41 & 80,10 & 73,40 & 81,77 & 62,04 & 81,77 & 68,23 \\
\hline Hayes & 81,24 & 100 & 81,24 & 42,65 & 81,27 & 27,54 & 81,81 & 20,29 & 81,24 & 24,82 & 81,24 & 23,32 \\
\hline Soyb. L & 85,40 & 100 & 84,26 & 35,65 & 84,65 & 42,98 & 84,95 & 23,25 & 85,40 & 2,51 & 85,40 & 4,49 \\
\hline Bridges & 57,85 & 100 & 57,84 & 62,57 & 55,84 & 31,58 & 54,00 & 31,55 & 57,85 & 25,62 & 57,85 & 36,10 \\
\hline Glass & 68,26 & 100 & 68,02 & 40,26 & 68,25 & 23,85 & 67,29 & 21,80 & 66,45 & 14,02 & 67,92 & 20,83 \\
\hline Iris & 93,30 & 100 & 93,30 & 25,98 & 92,54 & 21,32 & 92,67 & 21,37 & 93,30 & 9,22 & 93,30 & 6,86 \\
\hline Wine & 90,90 & 100 & 90,10 & 42,05 & 90,10 & 20,45 & 90,49 & 20,73 & 89,01 & 15,46 & 90,90 & 25,26 \\
\hline Avg. & 79,17 & 100 & 78,60 & 58,17 & 77,87 & 41,05 & 77,66 & 33,79 & 78,30 & 25,82 & 78,64 & 28,79 \\
\hline
\end{tabular}

Table 2. Obtained results using different classifiers

\begin{tabular}{|c|c|c|c|c|c|c|c|c|c|c|c|c|}
\hline \multirow{3}{*}{ Datasets } & \multirow{2}{*}{\multicolumn{2}{|c|}{ i $A E S A$}} & \multirow{2}{*}{\multicolumn{2}{|c|}{$\begin{array}{c}\text { Probabilistic i } \\
\text { AESA }\end{array}$}} & \multirow{2}{*}{\multicolumn{2}{|c|}{ TLAESA }} & \multirow{2}{*}{\multicolumn{2}{|c|}{ ModTLAESA }} & \multicolumn{4}{|c|}{ PROPOSED CLASSIFIER } \\
\hline & & & & & & & & & \multicolumn{2}{|c|}{$\begin{array}{l}\text { Tree AEMD } \\
\text { (using DFS) }\end{array}$} & \multicolumn{2}{|c|}{$\begin{array}{c}\text { Tree AEMD } \\
\text { (using BFS) }\end{array}$} \\
\hline & $A C C$ & Comp & $A C C$ & Comp & $A C C$ & Comp & $A C C$ & Comp & $A C C$ & Comp & ACC & Comp \\
\hline Hep. & 81,03 & 52,8 & 80,64 & 32,44 & 81,33 & 87,54 & 81,66 & 72,65 & 80,51 & 14,6 & 80,01 & 13,9 \\
\hline Zoo & 96,0 & 19,4 & 94,00 & 17,51 & 96,00 & 42,74 & 96,00 & 23,95 & 95,65 & 9,26 & 94,22 & 8,35 \\
\hline Flag & 51,36 & 27,7 & 49,62 & 26,41 & 52,84 & 48,41 & 52,09 & 32,95 & 52,64 & 10,2 & 52,35 & 9,46 \\
\hline Echo. & 81,05 & 63,6 & 80,06 & 63,08 & 81,77 & 71,58 & 82,44 & 44,62 & 81,77 & 13,1 & 81,40 & 14,9 \\
\hline Hayes & 80,77 & 17,6 & 80,07 & 16,74 & 80,54 & 46,42 & 81,06 & 24,05 & 80,95 & 11,3 & 80,34 & 12,4 \\
\hline Soyb. L & 85,4 & 1,96 & 82,15 & 2,04 & 85,40 & 47,51 & 85,40 & 16,85 & 84,57 & 2,40 & 81,51 & 2,94 \\
\hline Bridges & 57,85 & 25,1 & 56,95 & 25,06 & 56,74 & 46,75 & 57,23 & 38,74 & 54,00 & 6,88 & 54,00 & 7,93 \\
\hline Glass & 66,34 & 12,6 & 66,21 & 12,06 & 67,92 & 62,47 & 67,72 & 22,85 & 67,37 & 8,33 & 65,54 & 7,53 \\
\hline Iris & 93,3 & 7,54 & 93,30 & 8,01 & 93,30 & 41,51 & 93,30 & 11,65 & 93,30 & 8,62 & 93,30 & 6,55 \\
\hline Wine & 90,01 & 10,6 & 90,90 & 10,54 & 90,90 & 39,75 & 90,90 & 12,64 & 90,90 & 9,26 & 90,10 & 4,09 \\
\hline Avg. & 78,31 & 23,9 & 77,39 & 21,39 & 78,67 & 53,47 & 78,78 & 30,10 & 78,17 & 9,39 & 77,28 & 8,81 \\
\hline
\end{tabular}




\section{Conclusions}

In this work, a fast approximate $k-M S N$ classifier for mixed data, based on a tree structure and an approximating-eliminating approach, not based on metric properties, was proposed. In order to compare our classifier, FN, MS, ONC, AESA, LAESA, iAESA, probabilistic iAESA, TLAESA, and ModTLAESA classifiers were implemented using the same prototype comparison function for mixed data. Based on our experimental results, it is possible to conclude that, the proposed classifier obtained a similar accuracy, but it needed the smallest number of prototype comparisons.

As future work, we are going to look for other elimination criteria, which could improve the performance of the proposed classifier.

\section{References}

1. Cover, T.M., Hart, P.E.: Nearest neighbor pattern classification. Trans. Information Theory 13, 21-27 (1967)

2. Vidal, P.E.: An algorithm for finding nearest neighbours in (approximately) constant average time complexity. Pattern Recognition Letters 4, 145-157 (1986)

3. Micó, L., Oncina, J., Vidal, E.: A new version of the nearest-neighbour approximating and eliminating search algorithm (AESA) with linear preprocessing-time and memory requirements. Pattern Recognition Letters 15, 9-17 (1994)

4. Figueroa, K., Chávez, E., Navarro, G., Paredes, R.: On the least cost for proximity searching in metric spaces. In: Àlvarez, C., Serna, M. (eds.) WEA 2006. LNCS, vol. 4007, pp. 279-290. Springer, Heidelberg (2006)

5. Mico, L., Oncina, J., Carrasco, R.: A fast Branch and Bound nearest neighbor classifier in metric spaces. Pattern Recognition Letters 17, 731-739 (1996)

6. Tokoro, K., Yamaguchi, K., Masuda, S.: Improvements of TLAESA nearest neighbor search and extension to approximation search. In: ACSC 2006: Proceedings of the 29th Australian Computer Science Conference, pp. 77-83 (2006)

7. Fukunaga, K., Narendra, P.: A branch and bound algorithm for computing k-nearest neighbors. IEEE Trans. Comput. 24, 743-750 (1975)

8. Moreno-Seco, F., Mico, L., Oncina, J.: Approximate Nearest Neighbor Search with the Fukunaga and Narendra Algorithm and its Application to Chromosome Classification. In: Sanfeliu, A., Ruiz-Shulcloper, J. (eds.) CIARP 2003. LNCS, vol. 2905, pp. 322-328. Springer, Heidelberg (2003)

9. Oncina, J., Thollard, F., Gómez-Ballester, E., Micó, L., Moreno-Seco, F.: A Tabular Pruning Rule in Tree-Based Fast Nearest Neighbor Search Algorithms. In: Martí, J., Benedí, J.M., Mendonça, A.M., Serrat, J. (eds.) IbPRIA 2007. LNCS, vol. 4478, pp. 306-313. Springer, Heidelberg (2007)

10. García-Serrano, J.R., Martínez-Trinidad, J.F.: Extension to C-Means Algorithm for the use of Similarity Functions. In: Żytkow, J.M., Rauch, J. (eds.) PKDD 1999. LNCS (LNAI), vol. 1704, pp. 354-359. Springer, Heidelberg (1999)

11. Hernández-Rodríguez, S., Martínez-Trinidad, J., Carrasco-Ochoa, A.: Fast k Most Similar Neighbor Classifier for Mixed Data Based on a Tree Structure. In: Rueda, L., Mery, D., Kittler, J. (eds.) CIARP 2007. LNCS, vol. 4756, pp. 407-416. Springer, Heidelberg (2007)

12. Blake, C., Merz, C.U.: Repository of machine learning databases. Department of Information and Computer Science, University of California, Irvine, CA (1998), http: / /www.uci.edu/mlearn/databases / 